\section{Les insulines des invertébrés sèment le trouble}

Sophie Raisin, Sophie Pantalacci, Pierre Léopold

$>$ Des travaux récents ont démontré la forte conservation de la voie de réponse aux peptides de type insuline/IGF (insulin growth factor) depuis les invertébrés jusqu'aux mammifères. Les études génétiques menées sur la drosophile ou le ver C. elegans ont en particulier démontré le rôle de cette voie dans la croissance tissulaire et la longévité. Très récemment, une famille importante de peptides insuliniques a été découverte dans ces deux organismes, suggérant de nouvelles fonctions associées à ces peptides ainsi que l'existence, chez les mammifères, d'autres membres de cette famille encore à découvrir. <

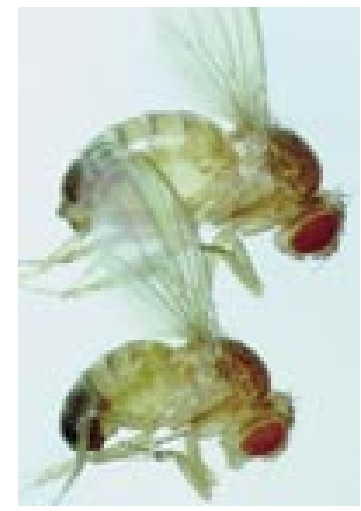

ceux-ci, les insulinlike growth factors (IGF-I et IGF-II) ont été caractérisés dans les cellules en culture comme des agents mitogènes qui contrôlent la survie et la prolifération cellulaires. Ils relaient dans les tissus l'effet de l'hormone de croissance (GH, growth hormone). Les IGF se lient à un récepteur cellulaire (IGF-IR) structurellement très proche du récepteur de l'insuline dont la signalisation intracellulaire - voisine de celle du récepteur de l'insuline - fait l'objet d'études détaillées du fait de son implication dans de nombreux cancers humains.

Les effets biologiques des peptides de type insuline passent par leur liaison aux deux chaînes $\alpha$ de leur récepteur, suivie de l'autophosphorylation des chaînes $\beta$. Cela augmente l'activité tyrosine-kinase du récepteur qui phosphoryle plusieurs protéines cytoplasmiques dont les IRS (insulin receptor substrate). II s'ensuit une cascade de phosphorylations par des kinases à sérine et à thréonine qui conduit à l'activation ou à la répression de substrats cellulaires (Figure 1).

Les voies de signalisation des peptides de la famille insuline, regroupées sous le terme générique de «signalisation insuline », ont été remarquablement conservées au cours de l'évolution. Ainsi, bien que l'analyse 
détaillée des mécanismes de transduction de ces différentes voies se poursuive avec succès dans les modèles cellulaires mammifères, le détour par des modèles génétiques puissants comme la drosophile et le nématode peut permettre de placer l'étude de ces voies dans le contexte d'un organisme entier et, à terme, grâce aux cribles génétiques mis en place dans ces organismes, de découvrir de nouveaux composants conservés de ces voies. Pour le moment, les études faites sur ces deux systèmes modèles ont déjà permis de mettre en évidence un rôle conservé de la signalisation insuline dans le contrôle de la croissance tissulaire, mais également de mettre à jour d'autres implications physiologiques de la voie, dans les phénomènes de longévité par exemple. En outre, des études très récentes sur les molécules de type insuline (appelées ILP pour insulin-like peptides) dans ces deux modèles pourraient changer notre façon de voir



Figure 1. Modèle de signalisation à partir des récepteurs de l'insuline/IGF.

La fixation d'un ligand de type ILP (insulin-like protein) sur les chaînes $\alpha \mathrm{du}$ récepteur conduit à l'autophosphorylation de tyrosines dans la partie cytoplasmique et à l'interaction des domaines PH /PTB de I'IRS (insulin receptor substrate) avec le récepteur. L'IRS est à son tour phosphorylé sur tyrosine et recrute des molécules à domaine SH2 comme la PI3-kinase accompagnée de sa sousunité régulatrice $\mathrm{p} 85$. La localisation de PI3K à la membrane permet de produire localement des phosphatidyl-inositol 3-phosphate (PtIns [3]P) et d'y ancrer les kinases PDKl (3-phosphoinositide-dependent protein kinase-1) et 2, puis AKT/PKB (protéine kinase $B$ ) et PKC $\zeta / \lambda$ situées en aval. L'activation par PDKI de la kinase PKB/AKT permet d'induire des réponses cellulaires aussi variées que le métabolisme du glucose via Gsk3 (glycogen synthase kinase 3), la survie cellulaire via Bad ou la machinerie de traduction des protéines via l'activation de la S6-kinase et du facteur d'initiation de la traduction elF4E. Alternativement, IRS ou SHC peuvent fixer l'adaptateur Dkr/Grb2 et déclencher l'activation de la cascade des MAP-kinases en réponse à l'activation du récepteur. cette famille de peptides et la fonction de ses membres chez les mammifères.

\section{Un rôle conservé de la signalisation insuline dans la croissance et le métabolisme}

Chez la drosophile, la croissance tissulaire

s'effectue au cours des trois stades larvaires. L'adulte naît des structures mises en place chez la larve avec une taille qui n'évoluera pas. Les pertes de fonction des gènes codant pour des composants conservés de la voie de signalisation insuline (dINR, Chico [IRS], dPI3K, dAKT, dPDKl, dS6K)* provoquent des réductions importantes de la masse des tissus larvaires et de la taille des organes et des appendices adultes, témoin d'un défaut important de croissance larvaire [1] $(\rightarrow)$. Dans la plupart des $(\rightarrow) \mathrm{m} / \mathrm{s}$ cas, l'analyse à l'échelle cellulaire des appen- $2000, n^{\circ} 1$, dices adultes révèle une réduction de la taille et p. 119 du nombre des cellules. A l'inverse, la surexpression du récepteur dINR à l'aide d'un transgène provoque une augmentation de la taille moyenne des cellules ainsi que de leur nombre. La surexpression de la PI3K (PI3 kinase) et des autres composants situés en aval conduit à une augmentation de la taille mais pas du nombre des cellules, indiquant que l'activation de la voie PI3K seule peut stimuler la croissance des cellules mais pas leur prolifération [2]. L'effet prolifératif du récepteur de l'insuline (INR) dans la croissance des tissus pourrait être relayé par l'IRS de drosophile (chico) dont les sites de fixation pour l'adaptateur Drk/Grb2 peut faire le lien vers d'autres voies comme celle des MAP-kinases. Enfin, les défauts de croissance des différents mutants de la voie insuline chez la drosophile sont évocateurs des phénotypes observés en situation de carence nutritionnelle, ce qui suggère que la signalisation insuline puisse être utilisée pour coupler les conditions nutritionnelles à la croissance des tissus.

Ce rôle de la voie insuline dans le contrôle de la croissance cellulaire est évidemment à rapprocher de celui des IGF de mammifères [3] et du fort retard de croissance associé à une mutation homozygote dans le domaine kinase du récepteur de l'insuline, retrouvé chez des patients atteints du syndrome de résistance insulinique aiguë [4]. En ce qui concerne le contrôle du métabolisme général, les mutations de l'IRS et du récepteur de l'insuline chez la drosophile provoquent également l'accumulation d'une grande quantité de lipides chez l'adulte $[5,6]$. Ce phénotype est à rapprocher de l'hypertriglycéridémie des souris mutantes pour IRSI, ou de l'accumulation de lipides chez les nématodes mutants pour le gène daf-2 codant pour le récepteur

\footnotetext{
* d : drosophile.
} 
de l'insuline. II démontre également l'existence d'une fonction conservée dans le métabolisme cellulaire des lipides $[7,8]$.

\section{Un rôle conservé dans la longévité}

Des mutations entraînant une forte réduction de l'activité de Daf-2, l'unique homologue chez le nématode du récepteur de l'insuline/IGF, induisent un arrêt du développement larvaire à un stade encapsulé dit dauer, qui s'accompagne d'un arrêt de croissance et d'une résistance au stress. Les mutations faibles dans le récepteur Daf-2 ne provoquent pas d'arrêt au stade dauer mais un doublement de la durée de vie normale des animaux $[8,9]$. De nombreuses études moléculaires et génétiques ont conduit à l'identification,

$(\rightarrow) \mathrm{m} / \mathrm{s}$ $2001 n^{\circ} 6 / 7$, p. 767 la longévité provoquée par la baisse de fonction daf-2 nécessite un facteur de transcription de la famille Forkhead/winged helix, daf-16, dont les homologues vertébrés AFX, FKHR et FKHRLl sont maintenant à l'étude : ces facteurs sont effectivement sensibles à l'activation de la voie insuline/IGF qui peut bloquer leur entrée dans le noyau et leur fonction transactivatrice [11, 12]. Enfin, les études d'individus mosaïques mutants pour daf-2 chez le ver indiquent que le rôle de daf-2 dans la longévité implique un nombre limité de neurones capables de relayer le signal via l'expression d'une deuxième molécule encore inconnue, sécrétée en direction du reste des cellules de l'organisme.

L'hypothèse d'une implication de cette voie de signalisation dans le contrôle de la longévité a été récemment confortée chez les insectes. $\varepsilon$ n effet, chez la drosophile, les mutants nuls chico (IRS) sont viables, de taille réduite, et leur durée de vie moyenne est allongée de $48 \%$. Cet effet ne semble pas lié à la réduction de la taille des individus homozygotes, puisque l'on retrouve un allongement de la durée de vie de $36 \%$ chez les hétérozygotes, qui, eux, sont de taille normale [13]. De même, certaines combinaisons d'allèles « perte de fonction pour le récepteur dINR » sont associées à des extensions de la durée de vie de $85 \%$ [14]. Dans ce cas, il a été montré que la perte de fonction dINR induit une chute de la sécrétion d'hormone juvénile dans le cerveau. Cette hormone rétinoïde, proche fonctionnellement et structurellement des thyroxines de mammifères, voit normalement sa sécrétion chuter lors de l'induction de la diapause* chez les insectes, qui se traduit par un arrêt des fonctions reproductives, une résistance au stress et une survie prolongée. L'extension de la longévité des mutants dINR pourrait donc être un effet indirect de la chute de sécrétion d'hormone juvénile chez ces animaux.

Chez la drosophile comme chez le ver, la voie InR semble

* Arrêt du développement au cours de la vie d'un insecte. donc impliquée dans la longévité par son action sur des tissus endocrines spécialisés, pour coordonner de manière simple le vieillissement des différents tissus dans l'organisme. Ces effets sont à mettre en parallèle avec la longévité accrue (+ 45-65\%) associée à une forte réduction de la sécrétion de GH, d'IGF-I et de TSH (thyroid stimulating hormone, hormone thyréotrope) des lignées de souris naines Snell et Ames [15].

Si les récepteurs et les voies de l'insuline sont conservés, il semblait logique de retrouver également des ligands capables de transmettre l'information. La découverte d'ILP chez la drosophile et le nématode fait l'objet de publications récentes. Une première surprise a été de trouver un grand nombre de ces molécules au regard de I'unique récepteur connu dans ces deux organismes. De plus, certains de ces ligands ont une action antagoniste sur l'activation du récepteur.

\section{Sept insulin-like proteins chez la drosophile...}

On sait que, chez les mammifères, les ILP sont synthétisées sous la forme de précurseurs dont la maturation s'effectue par clivage : deux des peptides produits, appelés $A$ et $B$, reliés par des ponts disulfures conservés, forment la molécule active (Figure 3). Afin d'identifier des potentiels ILP à partir des banques de données provenant du séquençage complet du génome de la drosophile, les chercheurs ont utilisé des critères de conservation des espacements entre les cystéines du domaine A participant à la formation des ponts disulfures. Sept ligands potentiels du récepteur de

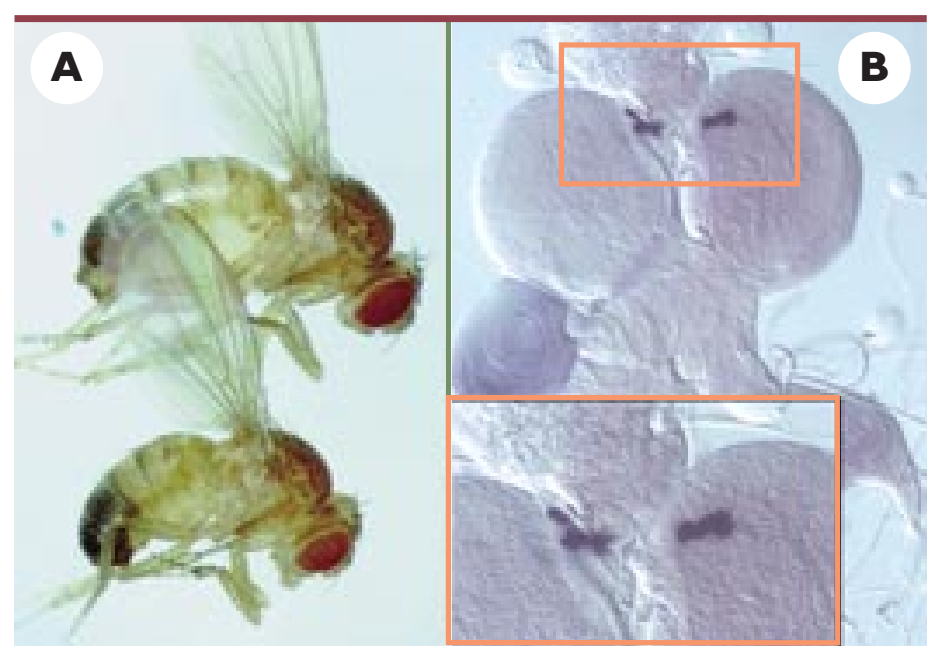

Figure 2. Expression de DILP2 chez la drosophile. A. L'expression ubiquitaire du gène DILP2 chez la larve provoque une augmentation proportionnée de la taille et du poids (+39\%) de l'adulte (haut), en comparaison d'un animal témoin (bas) (d'après [6]). B. Le gène DILP2 est exprimé au sein d'un groupe de sept cellules neurosécrétrices dans chacun des deux lobes cérébraux chez la larve. Les mêmes sept cellules expriment aussi les gènes DILP3 et DILP5 (d'après [6]). 


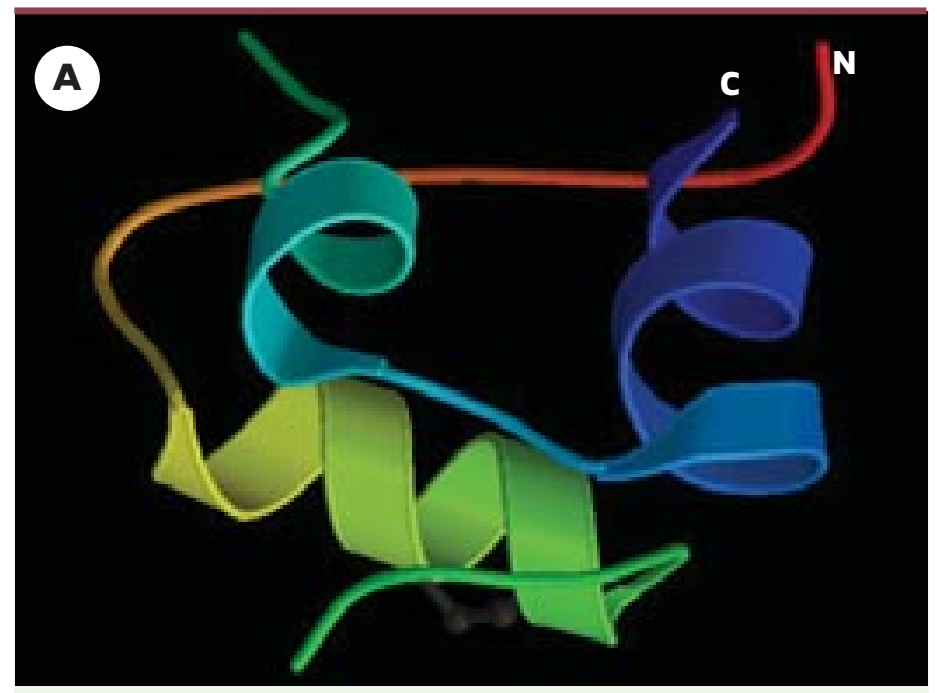

B

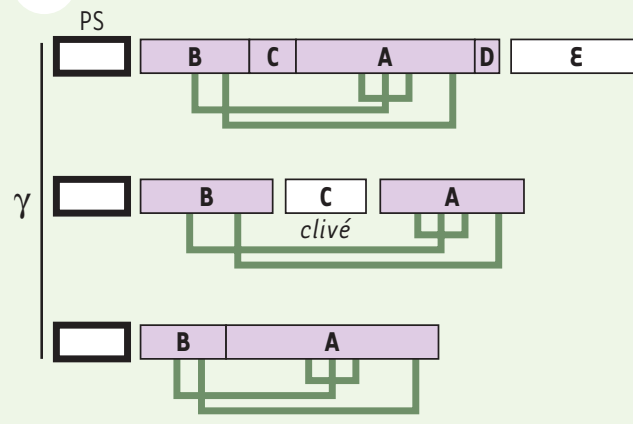

IGF, ins 11-17

insuline, DILPI-7, ins 18

ins 19,32 et 37

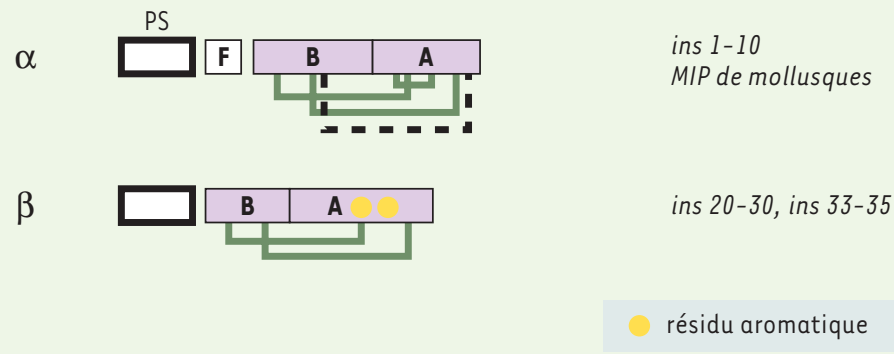

Figure 3. Conservation et diversité des homologues de l'insuline. A. Représentation tridimensionnelle de la molécule d'insuline humaine. « $\mathrm{N} \gg$ correspond à l'extrémité $\mathrm{N}$-terminale de la chaîne $\mathrm{B}$ et « $\mathrm{C} \gg$ à l'extremité $\mathrm{C}$-terminale de la chaîne $A$. On note la présence d'une hélice $\alpha$ dans la chaîne $B$ et de deux hélices $\alpha$ reliées par une boucle dans la chaîne $A$. $B$. Les homologues mammifères de l'insuline, les DILP de drosophile et un tiers des ins de nématode font partie de la même sous-classe $\gamma$. Trois ponts disulfures sont prédits qui relient les chaînes $A$ et $B$ et stabilisent la structure tridimensionnelle des peptides. Les différences se situent sur le peptide $C$, présent ou pas dans la séquence du précurseur et clivé ou pas dans le produit mûr. Les autres ins de $C$. elegans révèlent une plus grande diversité d'organisation et créent deux classes supplémentaires $\alpha$ et $\beta$. La famille $\beta$ est caractérisée par la présence de 4 ponts disulfures et d'un peptide F clivé. Elle comprend aussi les MIP, homologues de l'insuline chez les mollusques. La famille $\alpha$ ne possède que 2 ponts disulfures : deux cystéines de la chaîne A sont remplacées par des résidus aromatiques dont l'interaction forte assure la même stabilisation que le pont intra-chaîne.
I'insuline, appelés DILP (Drosophila insulin-like peptides), ont été identifiés [6]. Les sept peptides trouvés sont plus proches de l'insuline que des IGF, avec notamment des sites de clivage consensus entre les domaines $B$ et $A$ permettant l'excision du peptide C intercalant (Figure 3). Le niveau d'homologie le plus élevé est de $37 \%$ entre DILP2 et l'insuline humaine. La surexpression du gène DILP2 chez la larve provoque une augmentation conséquente de la taille et du poids (+39\%) des adultes, caractérisée par une augmentation de la taille et du nombre des cellules dans les appendices comme l'aile ou l'œil (Figure 2A, [6]). Cet effet sur la croissance de l'organisme nécessite que le récepteur soit fonctionnellement intact : l'excès de croissance dû à la surexpression de DILP2 est supprimé chez des mouches hétérozygotes pour une mutation du récepteur dINR. Inversement, la surexpression du récepteur dans l'œil provoque un phénotype d'œil gros et « rugueux » qui n'apparaît pas si une copie des gènes DILPI à 5 , regroupés en complexe, est supprimée. Ces résultats démontrent de manière élégante que le gène DILP2 est capable d'interagir avec le récepteur dINR pour promouvoir la croissance tissulaire.

Si le rôle propre de la transduction du signal insuline dans la croissance des cellules a été clairement établi, on ignorait jusqu'à présent quelles pouvaient être les sources du signal. Plusieurs expériences récentes ont impliqué le corps gras (qui cumule certaines fonctions du foie et du tissu adipeux chez la larve) dans la production de facteurs de croissance en réponse à l'état nutritionnel. Pourtant, aucun des gènes $d I L P$ n'est exprimé au cours du développement dans cet organe. Cependant, une expression de certains dILP est détectée dans les tissus en croissance, ce qui rappelle l'expression ubiquitaire des IGF chez les mammifères.

De manière plus intéressante, trois dILP sont exprimés de façon très localisée dans un groupe de neurones de chacun des deux lobes cérébraux chez la larve (Figure 2B) [6, 16]. Une expression très proche d'un ILP, la bombyxine, a été décrite chez le ver à soie Bombyx mori. La bombyxine, produite dans le cerveau du ver à soie par un groupe de quatre cellules neurosécrétrices, est transportée le long des axones vers les corpora allata, un des organes sécréteurs du système nerveux central, d'où elle semble passer dans I'hémolymphe. Le rôle physiologique de cette insuline est encore mystérieux, mais des études récentes montrent qu'elle est libérée du cerveau dans l'hémolymphe en réponse à la prise de nourriture. Inversement, en condition de jeûne, l'hormone s'accumule dans le cerveau et les concentrations circulantes dans l'hémolymphe chutent. Cet effet peut être totalement aboli par une injection de glucose dans l'hémolymphe des larves, suggérant que, de manière très analogue à la régulation de la sécrétion d'in- 
suline des mammifères, la concentration de glucose circulant est le stimulus nutritionnel déclenchant la libération de l'hormone [17]. De manière comparable, l'expression des DILP dans le cerveau de la drosophile pourrait correspondre à un rôle neurosécréteur de ces peptides, permettant d'intégrer au niveau central des informations nutritionnelles.

Les profils d'expression complexes des différents DILP permettent dans tous les cas de conclure à une combinaison d'effets neurosécréteurs et autocrines dans le contrôle de la croissance de l'organisme. La dissection génétique de la fonction de ces gènes chez la drosophile permettra bientôt d'y voir plus clair.

\section{... et 37 insulin-like proteins chez le ver !}

Une même approche génétique a été menée chez le nématode, utilisant des critères plus complexes de conservation de structure au sein des peptides A et B. Elle a permis l'identification de 37 gènes codant pour des membres de la superfamille des ILP (ins-1 à-37). II s'agit d'un nombre étonnamment élevé comparé à celui observé chez les mammifères, en particulier chez l'homme où seuls sept ligands, mais plusieurs récepteurs, sont connus. Chez le nématode, la plupart de ces gènes codent pour des protéines assez éloignées des ILP de mammifères et de drosophile ; le plus souvent, le peptide $C$ séparant les peptides $B$ et $A$ est absent et le positionnement des ponts disulfures est différent (Figure 3).

Parmi ces 37 gènes, 15 ont fait l'objet d'études d'expression in situ en utilisant des gènes rapporteurs green fluorescent protein fusionnés aux régions 5'. L'expression est majoritaire dans le système nerveux, en particulier dans des neurones sensoriels impliqués dans la formation du stade dauer [18, 19]. Certains, comme ins-1, sont également exprimés faiblement dans des tissus périphériques (vulve, intestin, épiderme ou pharynx).

L'étude fonctionnelle de ins-1, qui possède la plus forte homologie avec l'insuline humaine (32\%), a réservé quelques surprises. Les mutations touchant le gène ins-1 n'ont pas d'effet sur la fonction de daf-2, ce qui peut s'expliquer par une redondance fonctionnelle au sein de cette famille de gènes. A l'inverse, la surexpression de ins-1 provoque l'arrêt au stade dauer des animaux transgéniques et accentue le phénotype des mutants hypomorphes daf-2. Cela évoque une action antagoniste de ins-1 sur son récepteur présumé daf-2 [18]. De façon encore plus paradoxale, la surexpression d'un autre ILP (ins-18) ou du gène codant pour l'insuline humaine sous le contrôle du promoteur ins1 , induit ce même effet antagoniste sur la fonction de daf2, suggérant que le mécanisme d'inhibition de la voie daf2 par les ILP est plus général.
Comment peut-on expliquer le rôle antagoniste de ces trois ligands sur le récepteur daf-2 ? II est peu vraisemblable qu'il s'agisse d'un processus évolutif propre au gène daf-2 de C. elegans, ayant conduit à un effet antagoniste de la liaison des ILP au récepteur. D'ailleurs, des arguments génétiques indiquent l'existence, chez le nématode, d'activités agonistes de daf-2 qui sont sécrétées par certains neurones de façon dépendante du $\mathrm{Ca}^{2+}$. II paraît plus probable que les trois ligands testés soient capables de se lier au récepteur sans pouvoir l'activer, jouant ainsi un rôle d'inhibiteur compétitif vis-à-vis d'autres ligands agonistes de la voie. On peut envisager, qu'en réponse à des conditions externes favorables (nutrition), les neurones connus pour intervenir dans la transformation dauer libèrent une combinaison de neuropeptides ILP capables d'activer le récepteur daf-2. L'activation de cette voie, en prévenant la transition vers le stade dauer, permettrait le développement normal du ver. Dans des conditions plus défavorables, la libération d'autres ligands antagonistes comme ins- 1 et ins-18 bloquerait cette voie de signalisation et provoquerait la transition vers le stade dauer et l'allongement de la durée de vie.

La conclusion à tirer de ces travaux pour l'homme est double. Le nombre très élevé de ligands de type insuline chez les invertébrés contraste avec l'existence de récepteurs uniques. La logique évolutive qui tend vers la diversité et la multiplication des gènes chez les vertébrés au regard des invertébrés est confirmée par l'existence d'un plus grand nombre de récepteurs chez les mammifères. Cette même logique suggère fortement qu'il existe encore de nombreux membres de la superfamille de l'insuline à découvrir chez l'homme. Par ailleurs, le rôle antagoniste de certains ILP chez le ver suggère qu'un mécanisme de modulation des récepteurs de l'insuline ou des IGF par des ligands antagonistes, bien qu'encore inconnus chez l'homme, puisse exister. L'interêt thérapeutique potentiel de telles molécules dans le traitement de certains cancers impliquant les récepteurs de l'IGF devrait stimuler pour les années à venir les travaux de recherche dans cette voie. $\diamond$

\section{SUMMARY}

Lessons from invertebrate insulin/IGF pathway

Recent studies have demonstrated that Insulin/IGF pathway is remarkably conserved from invertebrates to mammals. Genetic studies carried in Drosophila and C. elegans have involved this pathway in the control of organismal growth and ageing. More recently, a large family of insulinlike molecules has been identified in these two systems, which suggests that additional functions might be carried by some of them, and also that new mammalian insulinlike molecules still have to be discovered. $\diamond$ 


\section{RÉFÉRENCES}

1. Stocker H, Hafen E. Genetic control of cell size. Curr Opin Genet Dev 2000 ; 10 : 529-35.

2. Weinkove D, Neufeld TP, Twardzik T, Waterfield MD, Leevers SJ. Regulation of imaginal disc cell size, cell number and organ size by Drosophila class I(A) phosphoinositide 3-kinase and its adaptor. Curr Biol $1999 ; 9: 1019-29$.

3. Lamothe B, Baudry A, Desbois $P$, et al. Genetic engineering in mice: impact on insulin signalling and action. Biochem / 1998 ; 335 : 193-204.

4. Takahashi Y, Kadowaki H, Momomura K, et al. A homozygous kinase-defective mutation in the insulin receptor gene in a patient with leprechaunism. Diabetologia $1997 ; 40$ : 412-20.

5. Bohni R, Riesgo-Escovar J, Oldham $S$, et al. Autonomous control of cell and organ size by CHICO, a Drosophila homolog of vertebrate IRSI-
4. Cell 1999 ; 97 : 865-75.

6. Brogiolo W, Stocker H, Ikeya $\mathrm{T}$, Rintelen $\mathrm{F}$, Fernandez $\mathrm{R}$, Hafen $\varepsilon$. An evolutionarily conserved function of the Drosophila insulin receptor and insulin-like peptides in growth control. Curr Biol 2001 ; 11 : 213-21.

7. Abe H, Yamada N, Kamata K, et al. Hypertension, hypertriglyceridemia, and impaired endothelium-dependent vascular relaxation in mice lacking insulin receptor substrate-1. J Clin Invest 1998 ; $101: 1784-8$

8. Kimura KD, Tissenbaum HA, Liu Y, Ruvkun G. daf-2, an insulin receptor-like gene that regulates longevity and diapause in Caenorhabditis elegans. Science 1997 ; 277 : 942-6.

9. Kenyon C, Chang J, Gensch E, Rudner A, Tabtiang R. A C. elegans mutant that lives twice as long as wild type. Nature 1993; 366 : 461-4.

10. Guarente L, Kenyon C. Genetic pathways that regulate ageing in model organisms. Nature 2000 ; 408 : 255-62.
11. Kops GJ, de Ruiter ND, De Vries-Smits AM, Powell DR, Bos JL, Burgering BM. Direct control of the Forkhead transcription factor AFX by protein kinase B. Nature $1999 ; 398: 630-4$.

12. Brunet $A$, Bonni A, Zigmond MJ, et al. Akt promotes cell survival by phosphorylating and inhibiting a Forkhead transcription factor. Cell 1999; $96: 857-68$.

13. Clancy DJ, Gems D, Harshman LG, et al. Extension of life-span by loss of CHICO, a Drosophila insulin receptor substrate protein. Science 2001 ; $292: 104-6$.

14. Tatar M, Kopelman A, Epstein D, Tu MP, Yin CM, Garofalo RS. A mutant Drosophila insulin receptor homolog that extends life-span and impairs neuroendocrine function. Science 2001 ; 292 : 107-10.

15. Bartke A, Brown-Borg H, Mattison J, Kinney B, Hauck $S$, Wright C. Prolonged longevity of hypopituitary dwarf mice. Exp Gerontol 2001 ; $36: 21-8$.
16. Cao C, Brown MR.

Localization of an insulinlike peptide in brains of two flies. Cell Tissue Res 2001 ; $304: 317-21$

17. Masumura M, Satake $S$, Saegusa H, Mizoguchi A. Glucose stimulates the release of bombyxin, an insulin-related peptide of the silkworm Bombyx mori. Gen Comp Endocrinol 2000 ; 118 : 393-9.

18. Pierce SB, Costa M, Wisotzkey $R$, et al. Regulation of DAF-2 receptor signaling by human insulin and ins-1, a member of the unusually large and diverse $C$. elegans insulin gene family. Genes Dev $2001 ; 15: 672-86$.

19. Bargmann Cl, Horvitz HR. Control of larval development by chemosensory neurons in Caenorhabditis elegans. Science 1991; $251: 1243-6$.
TIRÉS À PART

P. Léopold 